\title{
LABORATORY DIAGNOSIS OF MALARIA, A REVIEW
}

Raghuveer ${ }^{1}$. C. V, Mangala Goneppanavar².

1. Medical Director and Dean, Srinivas Institute of Medical Sciences and Research Institute, Mukka Surathkal, Mangalore

2. Assistant Professor, Department of Pathology, Mahatma Gandhi Medical College and Research Institute, Pillaiyarkuppam, Puducherry.

\section{CORRESPONDING AUTHOR}

Dr. Mangala G, Assistant Professor, Department of Pathology, MGMC\&RI, Pillaiyarkuppam, Puducherry, E-mail: mangalaanaghaa@gmail.com, Ph: 00919443987586.

ABSTRACT: BACKGROUND: The gross number of malaria cases is on the increase worldwide mainly due to limitations of traditional methods for malaria diagnosis. Even though peripheral smear examination of thick and thin film is widely accepted for detecting Plasmodium, species identification and for parasite count; it has its own pitfalls. Sensitivity of quantitative buffy coat is $41-93 \%$, specificity is $>93 \%$ for P. falciparum whereas $<52 \%$ for non-falciparum when parasitemia is $0.002 \%$. With availability of optimal reagents Immunochromatographic tests are being manufactured with different combination of target antigens to suit local malaria epidemiology. OptiMAL has high sensitivity compared to microscopy and ICT but low when compared to PCR. Serological tests are more useful for screening blood donor in case of transfusion- induced malaria with low parasitemia not detectable by blood film. PCR is used for detecting drug resistant strains, genotyping of Plasmodium strains. Placental histology is the best method for diagnosing malaria in pregnancy.

KEY WORDS: Laboratory, diagnosis, Malaria.

INTRODUCTION: Half of the world's population is at risk from malaria accounting for 250 million cases per year causing 8,60,000 deaths. ${ }^{1}$ Even though there is a downward trend in incidence of malaria in some countries, ${ }^{2}$ the gross number of malaria cases is still on the increase ${ }^{3}$ due to limitations of traditional methods. With emerging artemisinin-based combination therapies (ACT's) for malaria treatment, there is a need to look at efficacy and accuracy of various methods. ${ }^{4}$ Wherever laboratory support is not available, malaria diagnosis and treatment is done based on 'malaria score' and chances of making an accurate diagnosis in such cases are probably highest in P. vivax infections while lowest in P. falciparum. In high endemic areas fever is often equated with malaria and treated, which may be correct in some percentage of cases during transmission season, but not so outside that season. ${ }^{5}$ Contrary to this, there is misdiagnosis in non-endemic areas. Pitfalls of clinical diagnosis are i) over diagnosis of malaria ii) unnecessary morbidity and mortality as other illnesses due to underdiagnosis and undertreatment iii) over treatment and needless wastage of health resources spent on non-malaria cases iv) inappropriate prescription of anti-malarials contributing to anti-malarial drug resistance. ${ }^{6}$

Finding the parasite indicates infection, but does not necessarily imply disease. 'Threshold' above which infection generally means disease, is particularly difficult to define, 
because it is i)variable from one endemic area to another ii)age dependent and iii)needs to be adjusted to local level of endemicity.[7] Various methods for malaria diagnosis are:

I) Peripheral smear examination by light microscopy

II)Fluorescence microscopy techniques

III)Non-microscopic Rapid Diagnostic Tests

a) Immunochromatographic tests-detection of malaria antigen by HRP-2 and pLDH detection methods

b) Immunochromatographic dipstick assays used for diagnosis - ICT Pf, ParaSight $\mathrm{F}$ and

IV) Molecular methods.

\section{A) LIGHT MICROSCOPIC EXAMINATION OF PERIPHERAL SMEAR:}

Collection of blood sample: i) Before patients receive anti-malarial treatment ii) Minimum of three samples collected at heights of fever. Parasites can be concentrated by centrifugation because late trophozoites, schizonts and gametocytes get concentrated in the buffy coat and help in identifying P. vivax, P. malariae or P. ovale but not P. falciparum in case of low parasitaemia. THICK FILM: Touch the drop of blood with glass slide and spread it evenly with the corner of another slide, to make a square or circular patch of moderate thickness. THIN FILM: Blood drop should be smaller for thin film than for thick film. Stain peripheral smear with Leishman's or Giemsa, although Giemsa is preferred in the tropics. Field's is commonly used for staining thin film.

Giemsa stain: Examination of thick film: Before reporting a negative result, at least 200 oil immersion visual fields at 1000 magnification should be examined on both thick and thin smears, which have sensitivity of $90 \%$. A negative test DOES NOT rule out malaria.

\section{ADVANTAGES OF THICK FILM:}

1. Easy detection of parasites because of hemolysis

2. It is 20-40 times more sensitive than thin smear

3. Detection limit of 10-50 trophozoites/ul.

\section{DISADVANTAGES OF THICK FILM:}

1. Species identification not possible

2. Requires experience

INTERPRETATION OF THICK FILM: Young trophozoites - incomplete rings or spots of blue cytoplasm with a detached red chromatin dot. Late trophozoites of P. vivax - fragmented cytoplasm and Schuffner's stippling may be less obvious. Band forms of P. malariae are less characteristic but schizonts and gametocytes of these species retain their normal appearance, similarly gametocytes of P. falciparum also.

False positives:

1. Ghosts of hemolysed immature erythrocytes may be mistaken for Schuffner's stippling of P. vivax

2. Platelet clumps may simulate P. vivax

Journal of Evolution of Medical and Dental Sciences/Volume1/ Issue4/October - 2012 Page 454 
3. Vegetable spores, yeast may look like malarial parasites

4. Bacteria may contaminate the Giemsa solution and interfere with identification of malarial parasites. ${ }^{5}$

\section{THIN SMEAR ADVANTAGES:}

1. Species identification

2. Quantify parasitaemia

3. Assess for the presence of trophozoites, schizonts, gametocytes and

4. Detecting malarial pigment in neutrophils and monocytes.

Warhurst and Williams (1996) have discussed various methods for quantifying malarial parasites in thick smears. 1) Count the number of parasites in $200 \mathrm{WBC}$ fields (asexual forms). Assuming that each microliter of blood contains 8000 WBC's, parasite count is multiplied by 40 to give the number of parasites / ul of blood. Since WBC count may vary by ten folds in malaria infected patients, accurate density determination requires repetition of WBC counts each time peripheral smear is made. ${ }^{5}$ 2) Make thick smear with known volume of blood (5ul), count all the parasites in the smear and divide by 5 to get number of parasites/ul. Counting the parasites per WBC's is tedious and impractical in very high parasitaemia, ${ }^{7}$ so parasitized red blood cells (RBC's) in thin blood films are counted as an alternative. The approximate number of parasites present in $1 \mathrm{ul}$ of blood can be calculated by assuming that $1 \mathrm{ul}$ of blood contains $5 \times 10^{6}$ RBC's, so $1 \%$ parasitemia will contain 1parasite/100 RBC and 50,000 parasites/ul of blood. ${ }^{8}$ Alternatively counting the number of parasites / $1000 \mathrm{RBC}$ 's or again counting the number of parasites per 200 WBC and multiplying by a conversion factor can be done. A conversion factor of 4-4.5 million RBC's/ul is used to convert to a value of parasites/ul. Technicians use the following scoring method for 500-600 times magnification.

$$
\begin{aligned}
& +\ldots .1-10 \text { parasites / } 100 \text { thick film fields } \\
& ++\ldots 11-100 \text { parasites } / 100 \text { thick film fields } \\
& ++\ldots . .1-10 \text { parasites/thick film field and } \\
& ++++\ldots .10 \text { parasites/thick film field. }{ }^{6}
\end{aligned}
$$

Routinely check blood sample once in $12 \mathrm{hrs}$ in falciparum malaria, to know the reduction in the number of parasites per ul of blood. In falciparum malaria parasitemia is $>50 \%$, whereas in non-falciparum it rarely exceeds $2 \% .1$. To know the severity of malaria 2 .To monitor the response to treatment and 3. To detect drug resistant falciparum malaria cases. Developmental stages of parasites help in assessing severity when falsely low parasite count occurs in falciparum due to sequestration of parasitized erythrocytes in tissue capillaries. Presence of more mature parasite forms ( $>20 \%$ of parasites as late trophozoites and schizonts) and $>5 \%$ of neutrophils containing malarial pigment indicates more advanced disease and worse prognosis. ${ }^{4}$

I B) FLUORESCENT MICROSCOPY: To enhance the detection of parasites in peripheral smears, certain fluorescent dyes like Acridine Orange (AO) and Benzothiocarboxypurine (BCP) having an affinity for parasite nucleus can be used, both get excited at $490 \mathrm{~nm}$ and exhibit apple green or yellow fluorescence. Rhodamine-123 is especially useful in assessing viable parasites because uptake relies on an intact working parasite membrane. QBC-II (Quantitative Buffy Coat) method 
combines an AO coated capillary tube and an internal float, to mechanically expand the buffy coat together with the separation of lighter parasitized RBC's by centrifugation. Platelets concentrated below this buffy coat appear in upper RBC layer or within layer of platelets and WBC. ${ }^{7}$ ADVANTAGES: 1.Rapid 2.Qualitative screening method: stains the different leucocytes and malarial parasites differently 3.By centrifugation large volume of blood is examined and Parasites are concentrated in narrow zone which makes visualization of parasites easier. DISADVANTAGES: 1 . Non-specific fluorescence of nucleic acids of all cells especially platelets and Howell-Jolly bodies leads to the misinterpretation. 2. Requires microcentrifuge, fluorescent attachment and expensive capillary tubes. 3. Inferior to Romanowsky in identification of malaria species or for accurate parasite count. ${ }^{5}$ When there are $<100$ parasites/ul $(0.002 \%$ parasitemia), sensitivity is $41-93 \%$, specificity is $>93 \%$ for P. falciparum where as for P. vivax and other nonP. falciparum it is $<52 \%$, especially during later stages of development because they become denser during this period and may be hidden in the mononuclear cell layer. ${ }^{7}$

PROCEDURE: Fill the QBC capillary with blood from EDTA stained end, wipe the outer surface, till blood flows to other end (AO stained end), repeat 10-15 times, hold horizontally so that column of blood moves away from AO stained end. Close this end with finger, plug other end with plastic closure, insert the float inside capillary using forceps, gently tap capillary so that flat moves down. Centrifuge in microcentrifuge at $12,000 \mathrm{rpm}$ for $6-8$ minutes, place in the groove of capillary holder. OBSERVATION: Using transmitted bright light, focus buffy coat under low power, and change light to epifluorescent to appreciate the red, yellow, green layers. Switch off fluorescent light, turn on the transmitted light. Put a drop of fluorescent oil on capillary, change objective to $60 \mathrm{x}$ and focus the granulocyte layer, move the holder sideways and appreciate other layers.

INTERPRETATION: Schizonts, gametocytes - black pigmented structures in lymphocyte layer. Without changing the focus turn on the epifluorescent light and turn off the transmitted light, observe cells in the following order from closed end. Distinctly colored red cells, yellow to orange granulocytes, green lymphocytes and yellow plasma. Ring forms appear dull green with or without an orange dot at one side. Rings of P. vivax are larger than rings of P. falciparum. Double rings are of P. falciparum. Platelets appear as bright green small compact structures. P. vivax amoeboid forms - green irregular structures, schizonts - dull green round structures. Gametocytes of P. vivax - dull green large round structures mimicking small lymphocytes. To confirm turn to transmitted light, if black pigments are seen in these structures they are parasites and if not then they are lymphocytes. Gametocytes of P. falciparum - banana shaped green structures with central yellowish green pigment. Sometimes ring forms are seen in plasma layer also. In old blood sample mosaic pattern of RBC's in background with orange to yellow pigmented parasites seen. If capillary is examined after 24 hours two distinct colors of ring form can be appreciated. ${ }^{9,10}$

BCP intensely stains the nuclei of P. falciparum in contrast to poor staining of RBC inclusions and leucocyte nuclei, with $>95 \%$ sensitivity and specificity for P. falciparum. ${ }^{11}$

II) NON-MICROSCOPIC RAPID DIAGNOSTIC TESTS: Assays based on detection of malaria antigen indicate current infection. Features of an ideal target antigen are: 1.It should be abundant in blood and other body fluids 2.Be malaria specific with no cross-reactions with other microorganisms 3.Should not persist after parasitemia disappears. Initial tests were based 
on antigen-capture or antigen-competition format eg: ELISA After optimal reagents (i.e. monoclonal or polyclonal antibodies against specific malaria antigens) became available assays were simplified for the field use. ${ }^{5}$

Immunochromatographic (ICT) assay is done on small volume of blood 5-15ul using monoclonal antibodies impregnated on a test strip. Colored test line is obtained in 5-20 minutes. ${ }^{12}$ Commercial tests are manufactured with different combination of target antigens to suit the local malaria epidemiology. ${ }^{13}$ WHO recommendations for RDT's: 1.Results obtained from these test devices should be at least as accurate as results derived from microscopy 2.Sensitivity should be above 95\% compared to microscopy 3.Sensitivity of detecting 100 parasites/ul should be $100 \%$ 4.Ability to distinguish viable parasites from parasite products like antigens or nucleic acids that are not associated with viable organisms 5.Able to predict treatment outcome or resistance to antimalarial drugs and 6 . Should be able to assess parasite density.14 Antigens released by parasitized cells include those specific to Plasmodium falciparum Histidine-Rich-Protein 2 (PfHRP-2), Plasmodium falciparum specific Lactate Dehydrogenase (Pf-pLDH), antigen specific to Plasmodium vivax (Pv-Pldh), antigens common to Plasmodium falciparum, Plasmodium vivax, Plasmodium ovale and Plasmodium malariae [Panspecies parasite lactate dehydrogenase (pan-pLDH)] and Plasmodium aldolase. ${ }^{7}$ It is located on parasite cytoplasm and on parasitized erythrocyte membrane being expressed throughout the erythrocytic cycle,6,15,16 but its concentration varies as parasite moves from ring stage to late trophozoite stage. ${ }^{17,18}$ Although predominantly seen in asexual stage, it can be seen in early P. falciparum gametocytes. ${ }^{18,19}$ Current RDT's utilise Lateral-Flow Immunochromatographic (Dipstick) technology.

PROCEDURE AND PRINCIPLE: Here sample migrates as liquid across the surface of nitrocellulose membrane by means of capillary action. ${ }^{15,20}$ Performance of final assay depends on source of the antigen used. Two types of antibodies, capture antibody and detection antibody are used, either of these can be monoclonal or polyclonal. Even if monoclonal antibodies are directed against same antigen, but if targeted for different epitopes of same antigen, it may exhibit quite different sensitivities and specificities ${ }^{21}$ The capture antibodies are bound to membrane in an immobile phase, called so because they are fixed antibodies serve to extract and bind parasite antigen from migrating liquid sample. Detection antibodies are conjugated to an indicator like gold chloride in a mobile phase. This detection antibody-indicator(conjugate)parasite antigen complex diffuses further across strip until it is bound to capture antibody reacts to another epitope of target antigen. Since capture antibody is in immobile phase and is fixed to narrow section of strip conjugate, signal is concentrated and becomes visible as cherryred or purple colored line, if targeted antigen is present in the clinical sample. The excess of detection antibody-conjugate that was not bound by antigen and capture antibody, moves further until it is bound to goat anti-mouse antibody thereby generating control line. False positive Pf HRP-2 assay results occur for schistosomiasis caused by Schistosoma mekongi and for rheumatoid factor ${ }^{22,23}$ Pf HRP-2 has been detected upto 4 weeks following clearance of parasitemia in patients and travellers. ${ }^{24}$ RDT's combine a control line with one or more antigen detecting test lines: those with single test line are named two-band RDT's they target HRP-2 and detect Plasmodium falciparum only, while those with two and three test lines are called three and four band RDT's respectively because they detect other malarial parasites also (P. vivax, P. ovale and P. malariae) by using HRP-2 and Aldolase combined or Plasmodium lactate dehydroghenase (pLDH) as targets. Meta analysis of 21 studies on non-immune travellers with 
suspected malaria revealed high sensitivity (88-99\%) and specificity (95-100\%) of HRP-2 tests for detecting P. falciparum. Positive 3-band HRP-2 test results detected malaria better than 2band HRP-2 results. Negative HRP- 2 based test results excluded malaria better than parasite lactate dehydrogenase based tests. ${ }^{25}$ Most of the false negative results occurred at low parasite densities ( $<100$ asexual parasites/ul or $<0.002 \%$ of red cells infected), while others occurred at high parasite densities as defined by WHO i.e. infection with $>5 \%$ red cells infected which are attributed to genetic variations of HRP-2, but Prozone effect (high dose-hook phenomenon) is also an alternative explanation. It is common in one-step immunoassays like agglutination tests, just dilution of the sample will correct this effect. ${ }^{26}$ Since, HRP-2 persists in patients' blood for weeks after successful treatment pLDH is more appropriate for treatment monitoring. ${ }^{27}$ Not all therapeutic regimens including chloroquine and quinine, are effective against eradicating gametocytes and sulfa-containing regimens may actually induce gametocytemia, ${ }^{28}$ persisting gametocytes produce $\mathrm{pLDH}$ and it may remain positive despite clearance of asexual parasite forms. ${ }^{29}$ However persisting HRP-2 is advantageous in detecting low-level fluctuating parasitemia in chronic malaria. ${ }^{30}$ Mahadev et al compared ICT Pf/Pv with microscopy which revealed 97\% sensitivity and 98.3\% specificity for P. falciparum with PPV and NPV of 78 and $99.8 \%$ respectively. Several studies done at national and international level have revealed comparable results. ${ }^{31}$ Rapid dipstick method (OptiMAL) has high sensitivity compared with microscopy and ICT malaria P. f./P.v. ${ }^{32}$ but sensitivity is low compared with PCR. ${ }^{33}$ Series of studies done in 2011 to determine how various monoclonal pLDH antibodies work in combination, emphasize use of different anti-pLDH antibody combinations to optimize immunochromatographic pLDH tests. ${ }^{34}$

\section{III) SEROLOGICAL TEST:}

A) Antibody detection-Indirect Fluorescence Antibody (IFA) and

B) Antigen detection-Immunochromatographic (ICT) Immunoassays.

USES:

i) Screening blood donors in case of transfusion-induced malaria where donor's parasitemia may be below detectable level of blood film.

ii) Patient with fever suspected of malaria whose repeated blood smears are negative

iii) Testing patients treated for malaria recently but whose diagnosis is doubtful. ${ }^{35}$

IFA test is based on the principle of detecting antibodies against asexual blood stage malaria parasites. Titre of $>1: 20$ are positive, $<1: 20$ are unconfirmed malaria and $>1: 200$ titre are classified as recent infections. It is simple, sensitive but time consuming, expensive fluorescent microscope and trained personnel are required. Specific antibodies are produced after 1-2 weeks of infection and may persist for months to years in semi-immune patients from endemic areas with frequent re-infection. But in non-immune patient treated for single infection antibody levels fall more rapidly and may be undetectable by 3-6 months. Reinfection or relapse leads to secondary response with high and rapid rise in antibody titres. ${ }^{36,37}$ False negative results can occur in early acute phase.

\section{IV) MOLECULAR METHODS:}

A) POLYMERASE CHAIN REACTION: Highly sensitive, specific, used for confirming uncertain species, detection of drug resistant markers and genotyping of Plasmodium strains. 
PCR can detect as few as 1-5 parasites/ul of blood compared with around 50-100 parasites/ul of blood by microscopy or RDT. Also widely used for detecting Plasmodium Knowlesi in humans. ${ }^{38,39}$ The 18S rRNA is used as gene target for differentiation of malarial species by nested PCR and reverse-transcription PCR. Other DNA targets like circumsporozoite protein gene is also evaluated for species-specific regions. ${ }^{40,41}$ The detection limit using rt-PCR is 50 parasites/ml of whole blood, which is approximately 200 times lower than the detection limit of light microscopic diagnosis. Real-time Nucleic acid sequence-based Amplification (rt-NASBA) detects parasite RNS and is faster than PCR. $.22,43$

Micrototal analysis systems (micro TAS) will be able to undertake all steps in analysis and applicable for environments that lack infrastructure. Having high sensitivity they accurately diagnose mixed infections, detect drug- resistant strains and make other genetic markers available. 44

B) LAMP TECHNIQUE: Detects $18 \mathrm{~S}$ ribosome RNA of Plasmodium falciparum. It is easy, sensitive, quick and cheaper than PCR. However reagents require cold storage. ${ }^{45}$

C) FLOW CYTOMETRY: accurate, precise and rapid method with application in malaria research. ${ }^{46}$

D) MASS SPECTROPHOTOMETRY: is based on principle of detecting heme from hemozoin which is a parasite-specific biomarker and has sensitivity of detecting upto10 parasites /ul of blood. It requires $<1$ minute, automated but not suitable for rural areas where electricity is a problem. ${ }^{47,48}$

V) POST-MORTEM DIAGNOSIS OF MALARIA: ICT is sensitive, specific, used especially for nonimmune cases and also applicable in autolysed cases where microscopy is not possible. PCR can be used as confirmatory test, however one has to rely on histopathologic findings for malaria diagnosis in highly endemic areas where asymptomatic parasitemia is common. ${ }^{49}$ Increased presence of malaria hemozoin pigment in peripheral phagocytes can be used to diagnose malaria in severe and fatal cases.

VI) DIAGNOSIS OF MALARIA IN PREGNANCY: Studies have compared results of peripheral blood film, placental blood film with placental histology for diagnosis of Plasmodium falciparum in pregnancy, which revealed that Placental histology is more sensitive (91\%) than peripheral blood film (47\%) or placental blood film (63\%). So, placental histology is the best method for diagnosing malaria in pregnancy. 50

\section{REFERENCES:}

1. World Health Organization. WHO releases new malaria guidelines for treatment and procurement of Medicines. 9 March 2010. Press release.

2. James C, Sungano M, Philip T and Nirbhay K "A synopsis of current malaria diagnosis trends". Medical Journal of Zambia, 2009, vol 36 (2).

3. Breman JG, Alilio MS, and Mills A. Conquering the intolerable burden of malaria: What's new, what's needed: A summary. Am J. Trop. Med. Hyg 2004; 71(suppl 2): $1-15$. 
4. Kiszewski A, Teklehaimanot A. A review of the clinical and epidemiological burdens of epidemic malaria. Am J Trop Med Hyg 2004; 179 (suppl 2):128-135.

5. Marcel H. Diagnostic methods in malaria. In: Warrell DA and Gilles HM Eds. Essential malariology. $4^{\text {th }}$ Ed. New York: Arnold publishers; 2002 :35-58.

6. Collee JG, Mackie TJ and McCartney JE. Practical medical microbiology. $14^{\text {th }}$ Ed. New York. Churchill Livingstone. 1996.

7. McKenzie et al.WBC counts and malaria. J Infect Dis, 2005; 192:323-330.

8. Moody A. Rapid diagnostic tests in malaria. Clinical Microbiology Reviews. January 2002, 66-78 p.15(1).

9. Wongsrichanalai $\mathrm{C}$ et al. Acridine Orange fluorescent microscopy and the detection of malaria in populations with low-density parasitemia. Am.J.Trop.Med.Hyg.1991.44:17-20.

10. Briggs et al. Malaria detection using VCS technology. Am J Clin Pathol, 2006; 126:691-698.

11. Cooke A.H. et al. Use of fluorochrome benzothiocarboxypurine in malaria diagnosis. Trans.R.Soc.Trop.Med.Hyg. 1992.87:549.

12. Chansuda Wongsrichanalai et al. A review of Malaria diagnostic tools: Microscopy and Rapid diagnostic tests (RDT's). Am J Trop Med Hyg. 2007; 77(6):119-127.

13. Bell D, Wongsrichanalai C, Barnwell JW. Ensuring quality and access for malaria diagnosis: how can it be achieved? Nat Rev Microbiol. 2006; 4: 682-695.

14. World Health Organisation. 2000. WHO/MAL/2000.1091. New perspectives in malaria diagnosis. World Health Organisation, Geneva, Switzerland.

15. Howard R.J. et al.1986. Secretion of a malarial histidine -rich protein (Pf HRP II) from Plasmodium falciparum -infected erythrocytes. J Cell Biol. 103:1269-1277.

16. Shiff C.J., Premji Z, and Minjas J.N. 1993. The rapid manual ParaSight -F test. A new diagnostic tool for Plasmodium falciparum infection. Trans.R.Soc.Trop.Med.Hyg.87:646-648.

17. Rock E.P. et al.1987. Comparative analysis of the Plasmodium falciparum histidine-rich proteins HRP-I, HRP-II and HRP-III in malaria parasites of diverse origin. Parasitology 95:209-227.

18. Hayward R.E., Sullivan D.J. and Day K.P.2000.Plasmodium falciparum: histidine-rich protein II is expressed during gametocyte development.Exp.Parasitol.96:139-146.

19. Tjitra E. et al.2001.Persistent ICT malaria P.f/P.v panmalarial and HRP2antigen reactivity after treatment of Plasmodium falciparum malaria is associated with gametocytemia and results infalse positive diagnosis of Plasmodium vivax in convalescence.J.Clin.Microbiol.39:1025-1031.

20. Bell D and Peeling R.W.2006. Evaluation of rapid diagnostic tests: Malaria.Nat.Rev.Microbiol.4 (Suppl.9): S34-S38.

21. Lee $\mathrm{N}$ et al.2006. Effect of sequence variation in Plasmodium falciparum histidine-rich protein 2 on binding of specific monoclonal antibodies: implications for rapid diagnostic tests for malaria.J.Clin.Microbiol.44:2773-2778.

22. Eyal et al.2011.False positive Plasmodium falciparum Histidine-Rich protein 2 immunocapture assay results for Schistosomiasis caused by Schistosoma mekongi. Journal of Clinical Microbiology. 49(6):2331-2332.

23. Laferi H, Kandel K and Pichler H.1997.False positive dipstick test for malaria. N.Engl.J.Med.337:1635-1636.

24. Humar A et al.1997. ParaSight ${ }^{R} F$ test compared with the polymerase chain reaction and microscopy for the diagnosis of Plasmodium falciparum malaria in travelers.Am.J.Trop.Med.Hyg.56:44-48. 
25. Arthur M et al.2005.Meta-analysis:accuracy of rapid tests for malaria in travelers returning from endemic areas.Annals of Internal Medicine.142(10):836-846.

26. Philippe Gillet et al.2009. Assessment of prozone effect in Malaria Rapid Diagnostic Tests: Malaria Journal.8:271.

27. Moody A et al., 2000. Performance of the OptiMAL malaria antigen capture dipstick for malaria diagnosis and treatment monitoring at the hospital for Tropical Diseases, London. Br J Haematol 109:891-894. Cross RefMedlineWeb of Science

28. Murray C.K. et al., 2008. Update on rapid diagnostic testing for malaria. Clinical Microbiology Reviews. 21(1):97-110.

29. Miller RS, McDaniel $P$ and Wongsrichanalai C, 2001. Following course of malaria treatment by detecting parasite lactate dehydrogenase enzyme. Br J Haematol 113:558559. Cross Ref MedlineWeb of Science

30. Bell DR, Wilson DW and Martin LB, 2005. False-positive results of a Plasmodium falciparum histidine-rich protein 2-detecting malaria rapid diagnostic test due to sensitivity in a community with fluctuating low parasite density. Am J Trop Med Hyg 73:199-203.

31. Mahadev SH et al., Role of ICT malaria Immunochromatographic test for rapid diagnosis of malaria. J Pak Med Assoc 2006;56(4):167-171.

32. Khan SA et al., Comparison of optimal malarial test with light microscopy for diagnosis of malaria. J Pak Medical Assoc 2004; 54:404-407.

33. Iqbal J et al., Comparison of OptiMAL test with PCR for diagnosis of malaria in immigrants. J Clin Microbiology 1999;37: 3644-6.

34. Opportunities for improving pLDH-based malaria diagnostic tests (Malaria Journal),1 August,2011, My Journals.org

35. Igbinosa et al., A sequential review on accuracy of detecting malaria parasitemia in developing countries with restriction on resources. J. Med.Med.Sci.2010;1(9)385-390.

36. Garraud O, Mahanty S and Perraut R. Malaria specific-antibody subclasses in immune individuals: a key source of information for vaccine design. Trends Immunol.2003; 24:30-35.

37. Seed CR, Kitchen A and David TM. The current status and potential role of laboratory testing to prevent transfusion-transmitted malaria. Transfusion Med. Rev.2005; 19:225-240.

38. Cox-Singh J et al., Plasmodium knowlesi malaria in humans is widely distributed. Clin Infect Dis.2008;46: 165-171.

39.39) Luchavez J et al., Human infections with Plasmodium knowlesi, the Philippines. Emerg Infect Dis.2008;14: 811-813.

40. Snounou G et al., High sensitivity of detection of human malaria parasites by the use of nested polymerase chain reaction. Mol. Biochem. Parasitol. 1993; 61: 315-320.

41. Tahar R, Ringwald P and Basco LK. Diagnosis of Plasmodium malariae infection by the Polymerase chain reaction. Trans. R. Soc. Trop. Med and Hyg.1997; 91:410-411.

42. She RC et al., Comparison of immunofluorescence antibody testing and two enzyme immunoassays in the serologic diagnosis of malaria. J Travel Med 2007; 14:105-111.

43. Chotivanich K, Silamut K and Day NPJ. Laboratory diagnosis of malaria infection-a short review of methods. Aust J Med Sci 2006; 27:11-15. 
44. Peter G, Jutamaad S and Mathuros R. Microfluidic approaches to malaria detection. Acta Trop 2004; 89(3):357-369.

45. Han ET et al., Detection of four Plasmodium species by genus and speciesspecific loop mediated-isothermal amplification for clinical diagnosis. J Clin Microbiol 2007; 45:2521-2528.

46. Bhakdi SC et al., Re-evaluating acridine orange for rapid flow cytometric enumeration of parasitemia in malaria-infected rodents. Cytometry A. 2007; 71(9):643-5.

47. Scholl PF et al., Rapid detection of malaria infection in vivo by laser desorption mass spectrometry. Am J Trop Med Hyg.2004;71(5):546-51. 50)

48. Noppadan T et al., Malaria diagnosis: A brief review. Korean J Parasitol.2009; 47(2):93-102.

49. Nicole B. et al., Comparison of different methods for delayed post-mortem diagnosis of falciparum malaria. Malaria Journal 2009; 8:244.

50. Malaria diagnosis: Placental Histology best method for pregnant women. Malaria Weekly, June 2, 2003 